\title{
Human keratinocytes derived from the bulge region of hair follicles are refractory to differentiation
}

\author{
YUSUKE SASAHARA $^{1}$, YOSHIE YOSHIKAWA ${ }^{1}$, TOMONORI MORINAGA ${ }^{1}$, YOSHIRO NAKANO ${ }^{1}$, \\ NOZOMI KANAZAWA ${ }^{1,6}$, JOJI KOTANI ${ }^{2}$, SHIN KAWAMATA ${ }^{4}$, YOSHINOBU MURAKAMI ${ }^{4,7}$, \\ KATSUYUKI TAKEUCHI ${ }^{4}$, CHIZUKO INOUE $^{5}$, YUKIO KITANO ${ }^{3}$ and TOMOKO HASHIMOTO-TAMAOKI ${ }^{1}$ \\ Departments of ${ }^{1}$ Genetics, ${ }^{2}$ Emergency and Critical Care Medicine, Hyogo College of Medicine; \\ ${ }^{3}$ Hyogo College of Medicine, Nishinomiya; ${ }^{4}$ Foundation for Biomedical Research and Innovation, \\ Kobe, Hyogo; ${ }^{5}$ Department of Dermatology, Otemae Hospital, Osaka, Japan
}

Received December 16, 2008; Accepted February 13, 2009

DOI: 10.3892/ijo_00000247

\begin{abstract}
Human keratinocyte strains derived from the bulge region of plucked human follicles were successfully established from all 43 donors (age 24-76) regardless of the age and gender. The total cell number, number of population doublings and population doubling time were similar among the strains. These bulge-derived keratinocytes, BDKs, expressed keratin family genes specific to basal cell layers of the epidermis. They also expressed $C D 34$, one of the bulge stem cell marker genes. The growth behavior and positivity of CD34 indicate that BDKs contain stem cells. BDKs were cultured until confluency or treated with $\mathrm{CaCl}_{2}$ to induce differentiation. Morphology and expression of keratin family genes in BDKs before and after differentiation induction with $\mathrm{CaCl}_{2}$ were similar to those of epidermal keratinocytes obtained from skin biopsies (NHEKs). However, expression levels of keratin-10, a prickle cell layer marker, in $\mathrm{CaCl}_{2}$-treated $\mathrm{BDKs}$ were lower than those in $\mathrm{CaCl}_{2}$-treated NHEKs. Higher expression of integrin- $\alpha 6$, a basal cell layer marker, was also noted in BDKs than in NHEKs after differentiation induction. Expression of stem cell marker genes other than CD34, including CD200, Sox2 and NANOG, was about the same at confluency in both cells, but significantly higher in BDKs than NHEKs after differentiation. These results indicate that BDKs were more refractory to differentiation than NHEKs. We then examined Wnt signaling inhibitor genes, $D K K-3$ and $W I F-1$ that function
\end{abstract}

Correspondence to: Dr T. Hashimoto-Tamaoki, Department of Genetics, Hyogo College of Medicine, 1-1 Mukogawa-cho, Nishinomiya, Hyogo 663-8501, Japan

E-mail: tomokots@hyo-med.ac.jp

Present addresses: ${ }^{6}$ Department of Internal Medicine, Hyogo College of Medicine; ${ }^{7}$ Department of Indoor Environmental Medicine, Nara Medical University, Kashihara, Nara, Japan

Key words: bulge, hair follicle, stem cell, keratinocyte, Wnt signaling, human as tumor suppressors. $D K K-3$ expression decreased in both BDKs and NHEKs after $\mathrm{CaCl}_{2}$-induced differentiation. Expression of WIF-1 decreased $50 \%$ in BDKs one day after $\mathrm{CaCl}_{2}$ treatment and remained low, but was induced 1.7 times in NHEKs one day after $\mathrm{CaCl}_{2}$ treatment and further induced thereafter ( $>2.5$ times), suggesting that WIF-1 may be involved in maintaining the differentiation-refractory status of BDKs. Since cancer stem cells in the skin have been reported to be similar to bulge-derived stem cells, our BDK strains may be of use in studying characteristics of cancer stem cells of the epidermis.

\section{Introduction}

Epidermal stem cells were identified in the bulge region of hair follicles as label-retaining cells in mice, and their possible roles in skin carcinogenesis have been reported $(1,2)$. The mouse bulge region has been noted as a reservoir of multipotent stem cells that differentiate to epidermal keratinocytes and other skin appendices, such as sebaceous glands, hairs, and fat cells, and also cells of other lineage, such as neuronal cells, blood vessels and mesenchymal lineage cells (3-8).

Keratin-15 (K-15) has been noted as a marker of mouse and human bulge stem cells (9). CD34- and K-15-positive cells are label-retaining, characteristic of stem cells. Higher integlin- $\alpha 6$ (ITG- $\alpha 6$ ) expression is also noted in undifferentiated keratinocytes than in differentiated keratinocytes. CD34-positive cells show higher ITG- $\alpha 6$ expression than CD34-negative cells (10), showing that CD34 is one of the markers of bulge stem cells. The CD34-positive cells in the bulge region, separated by FACS, are round and relatively small in size, and grow rapidly in culture (10). CD34-positive and K-15-negative cells, present in the bulge region and considered to be more undifferentiated than CD34-positive and K-15-positive cells, can differentiate to neuronal cells and blood vessels $(5,6)$. These data indicate that the CD34positive cells existing in bulge regions are multipotent stem cells.

Human adult stem cells isolated from hair follicles, and neural crest and neuron stem cells express $N A N O G$ and 
OCT4 (Oct3/4). Oct3/4-positive cells were located in K-15positive area of the bulge region in vivo (11). Analysis of gene expression in K-15-positive bulge stem cells by cDNA microarray identify several bulge stem cell-specific genes, such as CD200, DKK-3, WIF-1, PHLDA1, follistatin, frizzled homolog 1, Sox2, Nanog and Oct-4 $(8,12,13)$. In humans, CD200 has been reported to be a stem cell marker more specific than CD34 (8). Among stem cell marker genes shown above, Oct3/4 and SOX2 were used to establish human iPS cells (14).

Undifferentiated keratinocytes in the basal cell layer of the epidermis divide and differentiate to the keratinocytes in prickle, granular, and keratinized cell layers. $\mathrm{CaCl}_{2}$ induces differentiation of epidermal keratinocytes in culture (15), with decreased expression of integlins- $\alpha 6$ and $-\beta 1$ and Keratins-5 and -14 (K-5 and K-14), markers of the basal cell layers, and increased expression of Keratins-1 and -10 (K-1 and K-10), markers of the prickle cell layers (5,16-18). However, there have been only a few reports on gene expression profiles of human bulge-derived keratinocytes in culture in relation to differentiation (8).

Bulge stem cells have been reported to play important roles in wound healing and skin cancer development. Implantation of mouse follicle cells into wound lesions of the skin result in recruitment of these follicle cells to cover wound lesions, but they are eliminated and replaced by basal cell layer-derived keratinocyte after the completion of wound healing, showing that follicle cells were not necessary for maintenance of the normal skin (19). However, molecular mechanisms of follicle cells in wound healing are not clear.

Basal cell carcinomas have been suggested to originate from the root sheath and/or the bulge region (20). CD34positive cells in mouse bulge regions were found to proliferate during multi-stage skin carcinogenesis (21). In the CD34knockout mice, basal cell carcinoma of the skin was not induced by treatment with chemical carcinogens (22), suggesting that CD34 is important to maintain cell proliferation and cancer formation. Recently, mouse early epidermal cancer cells were identified, which are similar to normal bulge stem cells phenotypically and functionally (23). These data suggest that the bulge region might be the reservoir of cancer stem cells as well as normal stem cells.

Skin biopsies are generally used to generate human epidermal keratinocyte cultures, but skin biopsy is highly invasive for donors. Plucked hair follicles can serve as an alternative source for epidermal keratinocytes in humans (24-26). In this study, we first established strains of human bulge-derived keratinocytes using plucked adult hair follicles and analyzed gene expression during $\mathrm{CaCl}_{2}$-induced differentiation in comparison to neonatal skin epidermis keratinocytes (NHEKs) derived from skin biopsy.

\section{Materials and methods}

Subjects. Adult 43 volunteers, 16 females of 24-55 years of age, and 27 males of 25-76 years of age, were enrolled in this study under informed consent. There was no significant difference in the average age between the female (39.6 \pm 5.2 years) and male $(39.5 \pm 6.1$ years) groups $(\mathrm{p}=0.97)$. Twenty of 43 donors (age $37.1 \pm 6.3$ years) had atopic features or atopic history including asthma and atopic dermatitis. This study was approved by the ethics board of Hyogo College of Medicine.

Primary cell culture from hair follicles. Hair follicles were plucked from occipital and temporal regions of each donor using forceps. Each hair was cut and the root portion containing hair follicles with the bulge region ( $\sim 5 \mathrm{~mm}$ long) was incubated in Dispase (Godo Shusei Co., Ltd., Tokyo, Japan) (500 U/ml in DMEM, Gibco/Invitrogen Co., NY, USA) at room temperature for $5 \mathrm{~min}$, placed in a $18 \mathrm{~mm}$-well (Becton-Dickinson Labware, NJ, USA) with $150 \mu 1$ of DKSFM medium (Gibco/Invitrogen), and incubated at $37^{\circ} \mathrm{C}$ under $5 \% \mathrm{CO}_{2}$. The medium was renewed every day.

Primary-outgrowth (PO) was observed usually within 7 days of culture. On day 14, the number of follicles with PO was determined and expressed as the percentage of the total follicles inoculated (PO rate). When cells growing from PO occupied nearly half of the well surface (day 10-14), cells were rinsed with trypsin-EDTA solution [0.05\% trypsin (Difco Lab, MI, USA) and $0.53 \mathrm{mM}$ EDTA (Gibco/BRL, MD, USA) in PBS(-)] twice, and incubated for $2 \mathrm{~min}$ at room temperature. Cells were then treated with trypsin inhibitor solution (Kurabo Industries Ltd., Osaka, Japan), gently suspended by pipeting, and centrifuged. Cell pellets were resuspended in DKSFM and transferred into a $60-\mathrm{mm}$ culture plate (Becton-Dickinson) (passage 1), and incubated with renewal of medium every 3 days. When cells reached $70 \%$ of confluency, they were trypsinized and $5 \times 10^{4}$ cells were transferred into a culture plate. Viable cells were counted to calculate the number and time of population doublings.

Storage of hair follicles before primary culture. Hair follicles were put into a $1.5 \mathrm{ml}$ cryotube (Iwaki, Tokyo, Japan) containing $1 \mathrm{ml}$ of DKSFM medium with or without HEPES (20 mM, Gibco/Invitrogen), and kept at RT or $4^{\circ} \mathrm{C}$ for $6 \mathrm{~h}$ with gentle shaking on a rocker platform $(10 \mathrm{rpm})$ before use.

Skin biopsy-derived keratinocytes. Primary cultures of neonatal foreskin-derived epidermal keratinocytes (NHEKs) in the first passage were obtained from Kurabo (Kurabo Industries). Five NHEK strains from different lots were used. Approximately 5000 cells were incubated in a $60 \mathrm{~mm}$ culture plate (passage 2) with DKSFM medium without HEPES. Cells were subcultured in the same manner as bulge-derived cells.

Induction of differentiation. Bulge-derived cells and NHEKs in 3-4 passages were placed in $100 \mathrm{~mm}$ plates $\left(1 \times 10^{5}\right.$ cells/ plate), and medium was renewed after two days. After 24-h incubation, cells reached in log-phase (day 0), and $1.2 \mathrm{mM}$ $\mathrm{CaCl}_{2}$ at a final concentration (Otsuka Pharm. Co., Ltd., Tokyo, Japan) was added. Medium was renewed on day 3.

$R T-P C R$. Isolation of total RNA and cDNA synthesis have been described (27). RT-PCR was performed using primers shown in Table I. PCR products were electrophoresed in agarose gels and the intensity of bands was measured by Adobe Photoshop CS2 (Adobe Systems, CA, USA). Gene expression levels were normalized by GAPDH. Real-time PCR was performed using Thermal Cycler Dice Real-Time 
Table I. Primers and PCR condition used for RT-PCR and real-time PCR.

\begin{tabular}{|c|c|c|c|c|}
\hline Gene & Forward primer & Reverse primer & $\begin{array}{l}\text { A.T. } \\
\left({ }^{\circ} \mathrm{C}\right)^{\mathrm{a}}\end{array}$ & Cycle \\
\hline Keratin $5(K-5)$ & 5'-GAC GTG GAG ATC GCC ACT TAC C-3' & 5'-AAA TTT GAC GCT GGA GCT GCT AC-3' & 63 & 27 \\
\hline Keratin $14(K-14)$ & 5'-GTG ACC ATG CAG AAC CTC AAC GA-3' & 5'-ACT CAT GCG CAG GTT CAA CTC TG-3' & 63 & 27 \\
\hline Keratin $1(K-1)$ & 5'-AGA TCT CCA ACT TGC AGC AGT CCA-3' & 5'-GGC TTG TGC TCA CAG ACA CAC TC-3' & 63 & 27 \\
\hline Keratin $10(K-10)$ & 5'-TGA CCG CCT GGC TTC CTA CT-3' & 5'-TCT GGC GCA GAG CTA CCT CA-3' & 63 & 27 \\
\hline Integrin $\alpha 6(I T G-\alpha 6)$ & 5'-GCG AGC CTT CAT TGA TGT GA-3' & 5'-TCA GAT GGC TGA GCA TGG AT-3' & 63 & 27 \\
\hline Integrin $\beta 1(I T G-\beta 1)$ & 5'-CAG CAA CGG ACA GAT CTG CA-3' & 5'-GAA CCA ACA GTC GTC AAC ATC-3' & 63 & 27 \\
\hline$C D 200$ & 5'-TCT CAC CCA AAT GGG ACC AC-3' & 5'-AGG GCT CTC GGT CCT GAT TC-3' & 63 & 33 \\
\hline CD34: first PCR & 5'-GCA TCT GCC TGG AGC AAA AT-3' & 5'-TGC AGC TGC ATG TGC AGA CT-3' & 62 & 20 \\
\hline CD34: nested PCR & 5'-AGG TAT GCT CCC TGC TCC TT-3' & 5'-AGC TCC AGC CTT TCT CCT GT-3' & 65 & Real-time \\
\hline Keratin $15(K-15)$ & 5'-AAG AAG TGG CCT CCA ACA CA-3' & 5'-TCT CGG CCA GTG AGT TCT CC-3' & 63 & Real-time \\
\hline$D K K-3$ & 5'-ACA GTG AGT GCT GTG GAG A-3' & 5'-TCT CTG GAA GGC ACA GCA CA-3' & 63 & Real-time \\
\hline$W I F-1$ & 5'-TGT CAA GAA GGT TGG CAT GG-3' & 5'-TTA AGT GAA GGC GTG TGC TG-3' & 64 & Real-time \\
\hline ATP5F1 & 5'-GCA GAA CAT GAT GCG TCG AA-3' & 5'-TGC ACT TGG CAA TTG TCT CC-3' & 63 & Real-time \\
\hline
\end{tabular}

${ }^{\mathrm{a} A . T}$, annealing temperature.

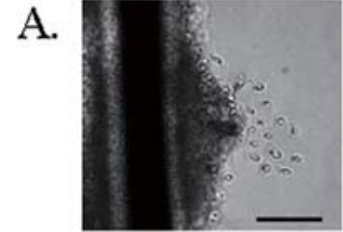

Day 3

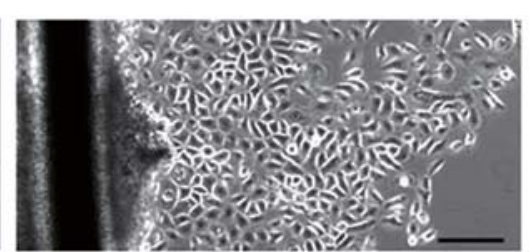

Day 7

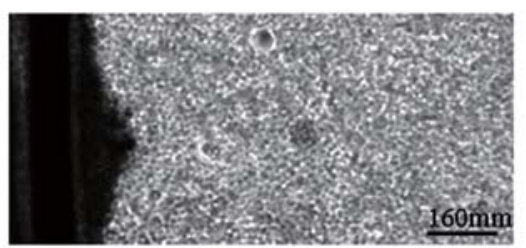

Day 14

B.

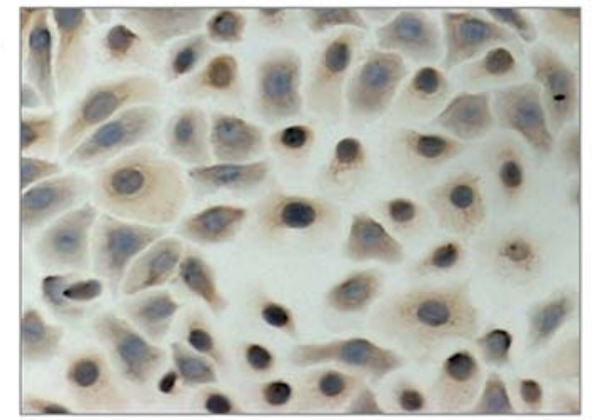

K-15

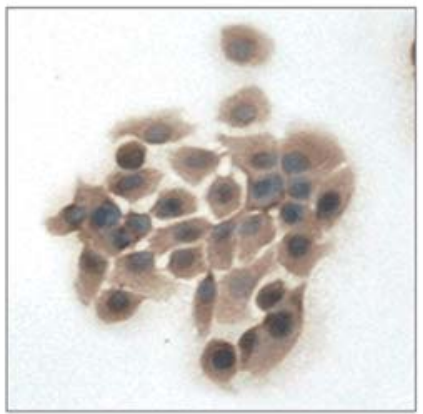

K-5

Figure 1. Morphology of primary outgrowth of a hair follicle. (A) Primary outgrowth cells from the bulge region of a hair follicle. Follicles were treated with Dispase, and incubated in an $18 \mathrm{~mm}$-well with DKSFM medium at $37^{\circ} \mathrm{C}$ (see Materials and methods). On day 3 , 20 cells were observed around the bulge region (primary outgrowth). On day 7 , cells were cobble stone-like in morphology. On day 14 , cells which were $~ 5000$ were trypsinized, and transferred into a plate (passage 1). (B) Cells in passage 1 were immunostained with antibodies against K-15 (a bulge cell marker) and K-5 (a basal cell layer marker). All cells were K-15- and K-5-positive.

System (Takara Bio Inc., Otsu, Japan) and gene expression levels were normalized by ATP $5 F 1$.

Immunostaining. Cells were fixed with paraformaldehyde. Monoclonal antibodies against CD34, K-5, K-10, and K-15 (Novocastra Lab. Ltd., UK) were used as the first and antimouse IgG1-HP (Nichirei Biosciences Inc., Tokyo, Japan) as second antibodies.

\section{Results}

Primary outgrowth. Three to 20 hairs were plucked from occipital and temporal regions of each donor, and hair follicles containing the bulge region were immediately inoculated in a well with DKSFM. After 3-7 days of incubation, 20 cells of cobble stone-like morphology were generated per bulge (primary-outgrowth, PO) (Fig. 1A). K-15, a bulge cell marker, 


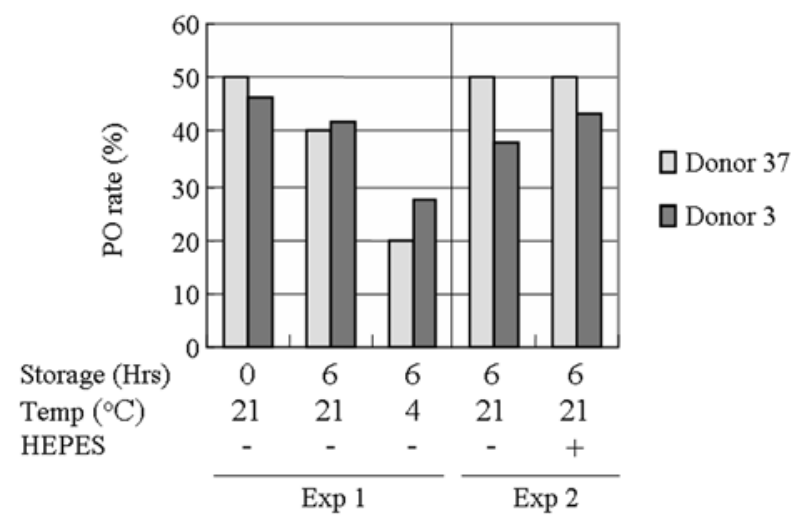

Figure 2. Storage condition after hair plucking and primary outgrowth rate. Hair follicles obtained from two donors (donor 37, a 26-year male and donor 3, a 55-year female) were soaked in DKSFM medium, transferred into a cryotube containing $1 \mathrm{ml}$ of DKSFM, and kept at room temperature or at $4^{\circ} \mathrm{C}$ before culture for $6 \mathrm{~h}$ with gentle shaking (exp. 1). The specimens were placed in culture wells. Primary-outgrowth (PO) was observed under a microscope on day 14 and the PO rate was calculated. The bulges were also kept in the DKSFM supplemented with or without HEPES $(20 \mathrm{mM})$ for $6 \mathrm{~h}$ with gentle shaking at room temperature before culture (exp. 2). Five to ten follicles were used for each experiment.

and K-5, a basal cell layer marker, were positive in $>90 \%$ of PO cells (Fig. 1B). The number of bulge-derived PO cells reached $\sim 5000$ after 10-14 days of incubation with forming a stratified structure (Fig. 1A). These cells, termed bulge-derived keratinocytes (BDKs), were also K-5 and K-15 positive (data not shown), but negative for CD34.

The effect of storage of hair follicles after hair plucking was examined using hair follicles from two donors (donor 37, a 26-year-old male, and donor 3, a 55-year-old female). The PO rate after $6 \mathrm{~h}$ of storage at room temperature was about the same as that of fresh used follicles immediately after hair plucking (Fig. 2). The PO rate decreased 50\% after $6 \mathrm{~h}$ of storage at $4{ }^{\circ} \mathrm{C}$. The addition of HEPES to the storage medium had little effect on the PO rate (Fig. 2). Based on these results, extracted hair follicles were immediately placed in DKSFM medium without HEPES, and used for culture within $6 \mathrm{~h}$.

The PO rate of 500 follicles obtained from 43 donors (24-76 years of age) ranged from 14 to $100 \%$ (average: $46.5 \%$ ) (Fig. 3). There was no significant difference in the PO rate between males and females $(49.0 \pm 5.2 \%$ vs $38.7 \pm 5.9 \%$, $\mathrm{p}=0.15)$, or among different age groups of every 10 years $(p=0.07)$. The PO rate in atopic donors did not differ significantly from that in non-atopic donors $(40.7 \pm 9.9 \%$ vs $49.8 \pm 5.9 \%, \mathrm{p}=0.07)$. PO was observed in two follicles out of three obtained from a 76-year-old male with alopecia areata, and the PO rate of a 66-year-old male was $\sim 50 \%$ (Fig. 3).

Cell growth and gene expression of BDK cells in sequential passages. Approximately 5000 cells were obtained from a follicle on day 14 of primary culture. These cells originated from each follicle (cell strain) were cultured separately. From five donors aged 26-66, more than five BDK strains were established. From each donor, five BDK strains were randomly selected and analyzed for cell growth. All 25 BDK strains from five donors showed similar growth. Growth

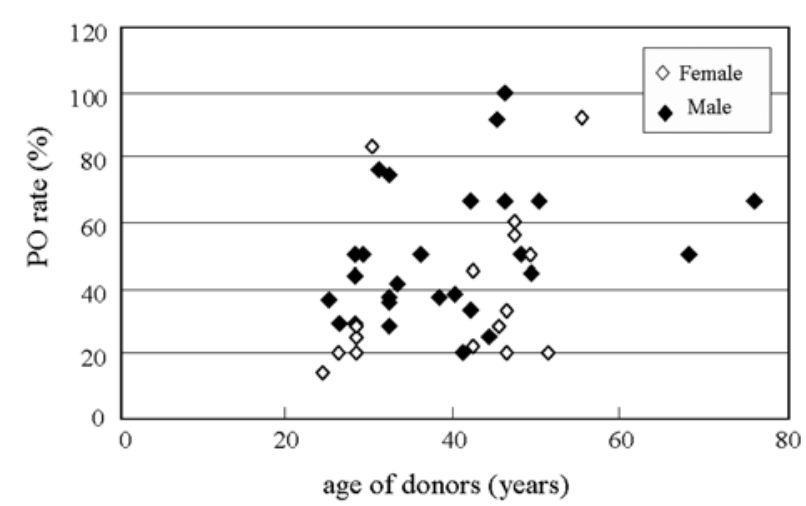

Figure 3. Primary outgrowth rate of all donors. BDK strains were established from 500 follicles from 43 donors. There were no significant differences in PO rate between male and female donors $(\mathrm{p}=0.15)$. There were no significant differences in age groups of every 10 years $(\mathrm{p}=0.07)$.

curves of cells from two donors, aged 66 and 26, are shown in Fig. 4A. All BDK strains could be cultured until 7-9 passages, and the number of population doublings was between 25 and 27. Between passages 2 and 4, the average population doubling time was $26.1 \mathrm{~h}$ (Table II). Total yields of BDKs from a follicle were $4.3 \times 10^{11}$ on average (Table II). These data showed that cell growth characteristics of BDK strains were similar and independent of the age of donor.

Five strains of NHEKs were used to study growth curves. Five thousand NHEKs were inoculated in a plate for the first passage. Growth curves of all NHEK strains were similar (Fig. 4A). The number of population doublings was less in BDKs than in NHEKs. The population doubling time between passages 2-4 was longer in BDKs than NHEKs. Total yield of BDKs was $\sim 1 / 10$ of NHEKs (Table II).

Three BDK strains obtained from three donors, a 26-yearold male, a 66-year-old male, and a 55-year-old female, and three NHEK strains were used for gene expression studies. In both BDKs and NHEKs, CD34 was detected in passages 2-4 (Fig. 4B) but hardly detectable at passage $4 . K-5$, a basal cell layer marker, was positive until passage $7 . K-10$, a prickle cell layer marker gene, was negative initially, but became positive in NHEKs and weakly positive in BDKs after passage 6. These results showed that, CD34-positive cells existed in cultured BDKs and NHEKs at least until passage 4. Spontaneous differentiation occurred after passage 4 but this process was slower in BDKs than NHEKs.

Comparison of gene expression between NHEK and BDKs. Treatment of BDKs with $1.2 \mathrm{mM} \mathrm{CaCl}_{2}$ caused flattening of cells and arrest of growth in 3 days. After 7 days of $\mathrm{CaCl}_{2}$ treatment, flattened and stratified cells with expression of K-10 were observed by immunostaining (Fig. 5). The growth arrest and morphological changes of BDKs after $\mathrm{CaCl}_{2}$ treatment were similar to those of NHEKs.

Gene expression was examined after treatment with $\mathrm{CaCl}_{2}$ in BDKs and NHEKs. $K-5$ and $K-14$ genes were expressed and $\mathrm{CaCl}_{2}$ treatment caused no significant changes in expression levels (Fig. 6A). In the absence of $\mathrm{CaCl}_{2}, K-1$ and $K-10$ were hardly detected in NHEKs and BDKs on 

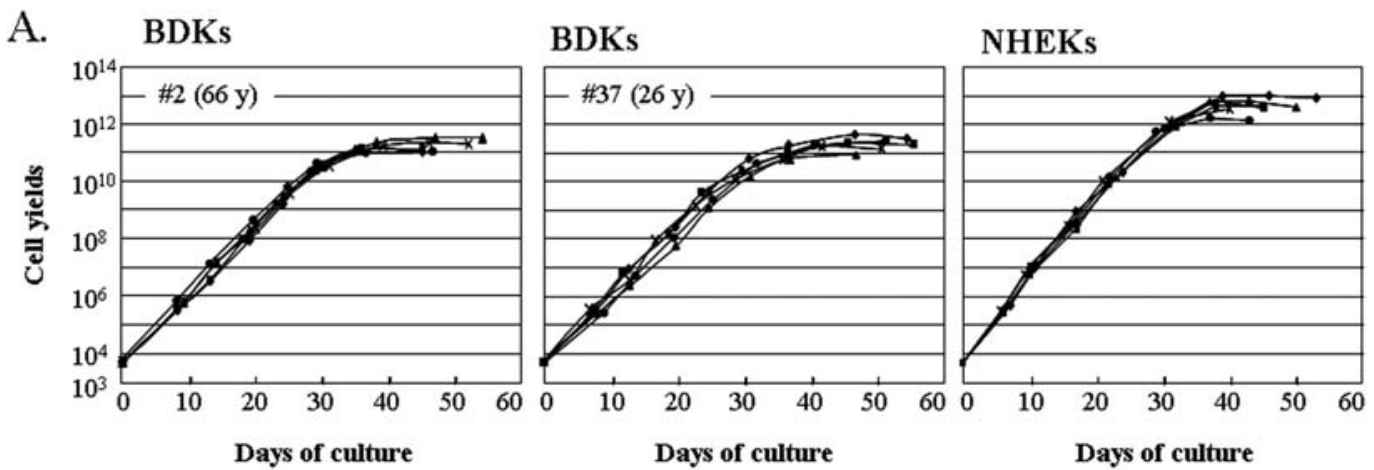

B.

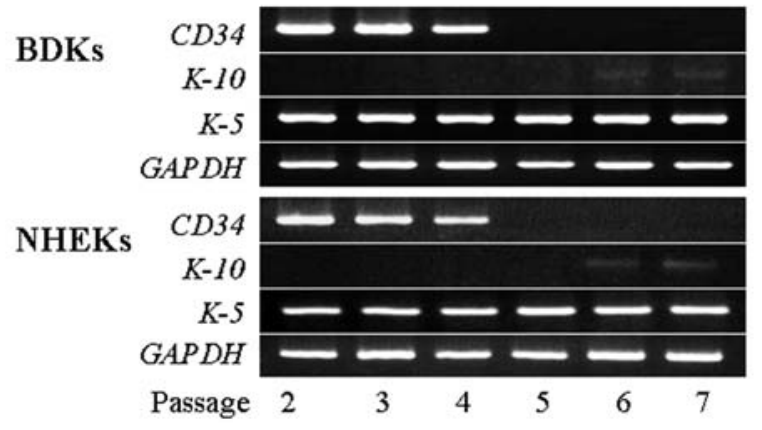

Figure 4. Cell growth and expression of the CD34, K-10 and K-5 genes. (A) Growth curves of BDK strains obtained from a 66-year-old male and a 26-year-old male are shown. From each donor, more than five BDK strains were obtained and five strains were randomly selected for growth assay. For NHEKs, five strains were used. At passage 1, all BDKs, 5000 , obtained from primary outgrowth were inoculated into a plate. Five thousand NHEKs in passage 1 were also inoculated in a plate. When BDKs and NHEKs reached $70 \%$ of confluency, cells were trypsinized and subcultured (passage 2). The points on the growth curves indicate passages. After passage 7, cells became flattened and large, resembling senescent cells. (B) Expression of the $C D 34, K$ - 10 and $K-5$ genes during passages was examined. Cells in a log-phase culture were harvested and gene expression was examined by RT-PCR (see Materials and methods). GAPDH was used for normalization of the expression level.

Table II. Number of population doublings (PDs), PD time and total cell yield of BDKs and NHEKs.

\begin{tabular}{lcccc}
\hline $\begin{array}{l}\text { BDKs } \\
\text { Donor no. }\end{array}$ & Age & No. of PDs & PD time (h) & Total cell yield $\left(\mathrm{x} 10^{11}\right)$ \\
\hline 2 & 68 & $26.5 \pm 1.0$ & $27.5 \pm 1.7$ & $4.5 \pm 3.1$ \\
3 & 55 & $26.3 \pm 1.0$ & $28.3 \pm 0.5$ & $4.1 \pm 2.1$ \\
12 & 46 & $25.5 \pm 1.0$ & $30.3 \pm 0.5$ & $1.7 \pm 0.9$ \\
26 & 33 & $27.0 \pm 1.4$ & $28.7 \pm 1.6$ & $9.4 \pm 8.5$ \\
37 & 28 & $25.5 \pm 1.0$ & $27.4 \pm 0.6$ & $1.8 \pm 2.6$ \\
Av. & & $26.1 \pm 1.1$ & $28.3 \pm 1.0$ & $4.3 \pm 6.9$ \\
\hline
\end{tabular}

\section{NHEKs}

strain no. Age No. of PDs PD time (h) ${ }^{\mathrm{a}} \quad$ Total cell yield $\left(\mathrm{x} 10^{11}\right)$

\begin{tabular}{ccccc}
\hline 1 & 0 & 31 & 25.1 & 110 \\
2 & 0 & 30 & 26.3 & 74 \\
3 & 0 & 29 & 25.1 & 49 \\
4 & 0 & 30 & 25.3 & 71 \\
5 & 0 & 28 & 25.5 & 10 \\
Av. & & $29.6 \pm 1.1$ & $25.5 \pm 0.5$ & $65 \pm 34$ \\
\hline
\end{tabular}

From each donor, five BDK strains established from five follicles were used for cell growth studies. Approximately 5000 BDKs from primary outgrowth of each follicle were inoculated into a culture plate at passage 1. Five NHEK strains were used and 5000 cells were also inoculated at passage 1 . Number of population doublings (PDs), PD time and total cell yield of each BDK and NHEK strains were calculated from growth curves. ${ }^{\text {aAverage time }}$ (hours) from 2 to 4 passages.

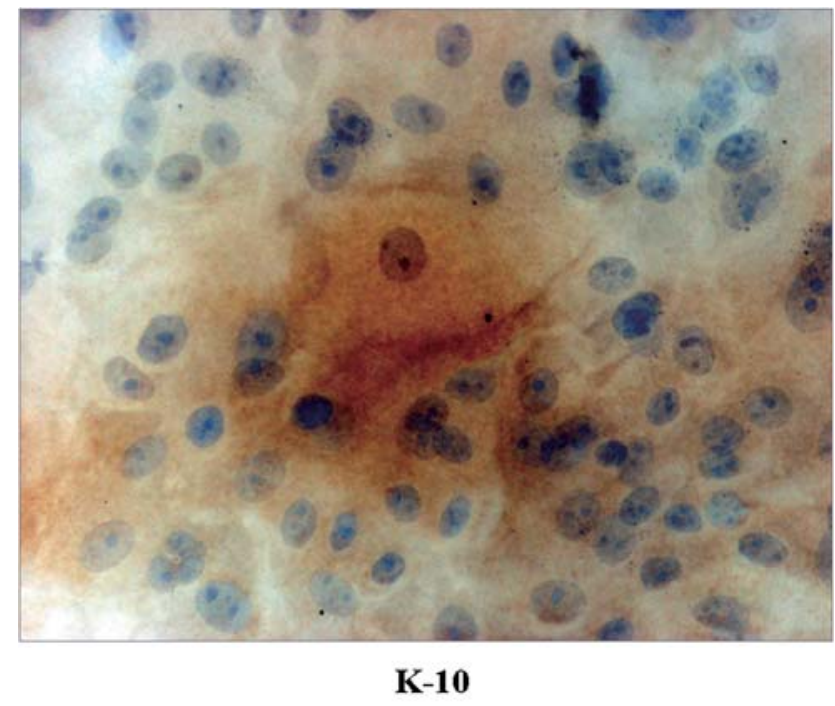

Figure 5. Expression of K-10 in BDKs after differentiation. Cells in passage 4 were inoculated in a plate and incubated for two days, then treated with $\mathrm{CaCl}_{2}(1.2 \mathrm{mM})$ for 7 days. Immunostaining with antibodies against K-10 (a prickle cell layer marker) showed flattened and stratified cells were $\mathrm{K}-10$ positive.

days 0 and 1 , became detectable on day 3 in NHEKs and on day 5 in BDKs and their expression levels further increased on day 7. In NHEKs with $\mathrm{CaCl}_{2}$ treatment, $K-1$ and $K-10$ became detectable on days 1 and 3 in BDKs. But levels of 

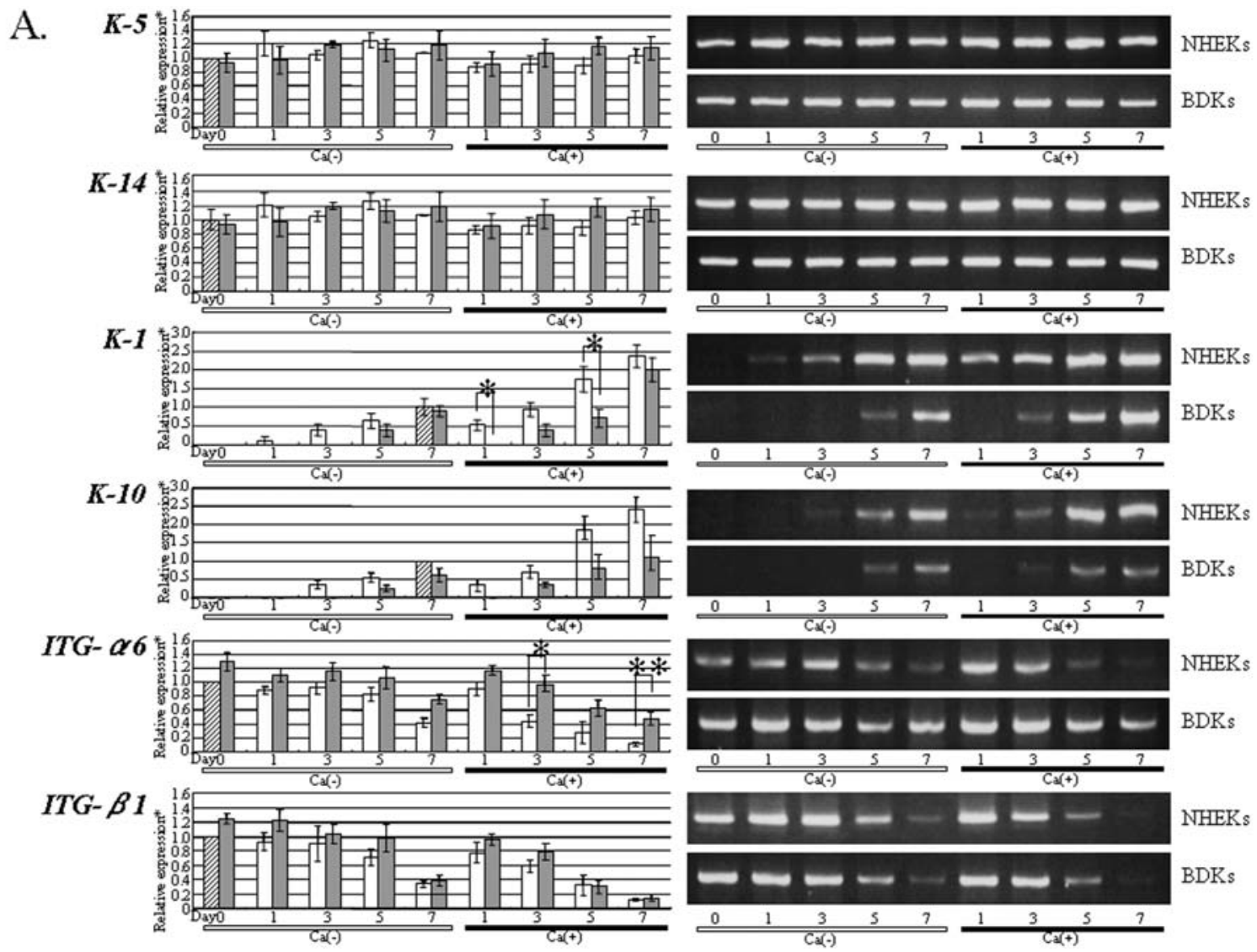

B.
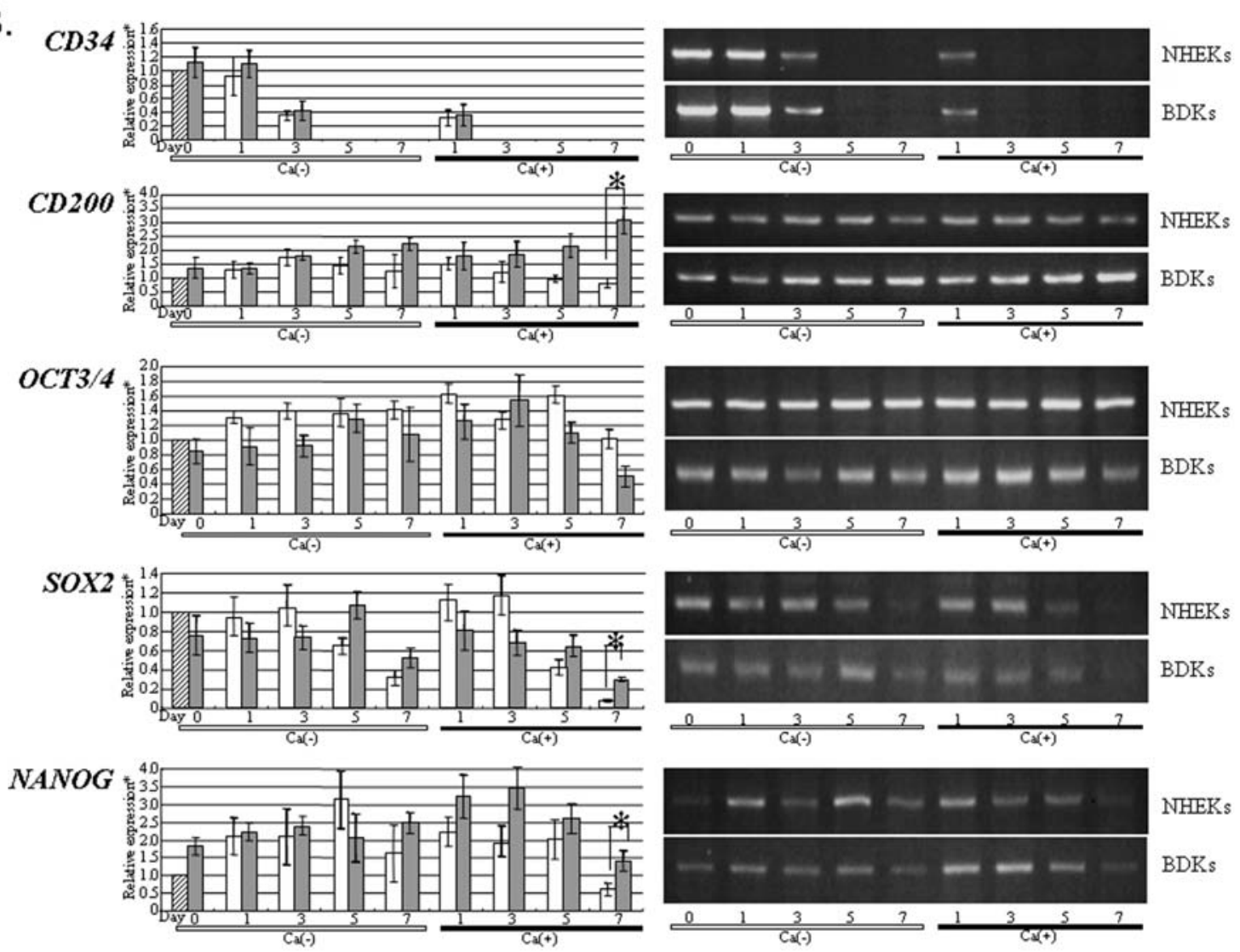

Figure 6. Expression of keratinocyte-specific genes and stem cell markers in BDKs and NHEKs during differentiation. Cells in passage 2-4, inoculated in a culture plate, incubated for two days and medium was renewed (day 0$)$. Cells were incubated with or without $\mathrm{CaCl}_{2}(1.2 \mathrm{mM})$. Cells were harvested on days $1,3,5$ and 7 , and analyzed for Keratins(K)-5, -14, -1 and -10, integrins (ITG)-a6 and - $\beta 1, C D 34, C D 200, O C T 3 / 4$, SOX2 and NANOG genes by RT-PCR (see Materials and methods). White and gray columns indicate NHEKs and BDKs, respectively. Expression levels were normalized to the levels of GAPDH. Expression levels of NHEKs on day 0 were set as 1.0 (shaded columns) except $K-1$ and -10. For $K-1$ and $K-10$, expression levels of NHEKs on day 7 were set as 1.0 (shaded columns). Each column shows the average of three independent experiments. Bar, I SD. (A) Expression of epidermal keratinocyte-specific genes. $K-5, K-14, I T G$ - $a 6$ and $I T G-\beta 1$ are basal cell layer markers, whereas $K-1$ and $K-10$ are prickle cell layer markers. (B) Expression of stem cell marker genes. ${ }^{*} \mathrm{p} \leq 0.05 .{ }^{* *} \mathrm{p} \leq 0.01$. 

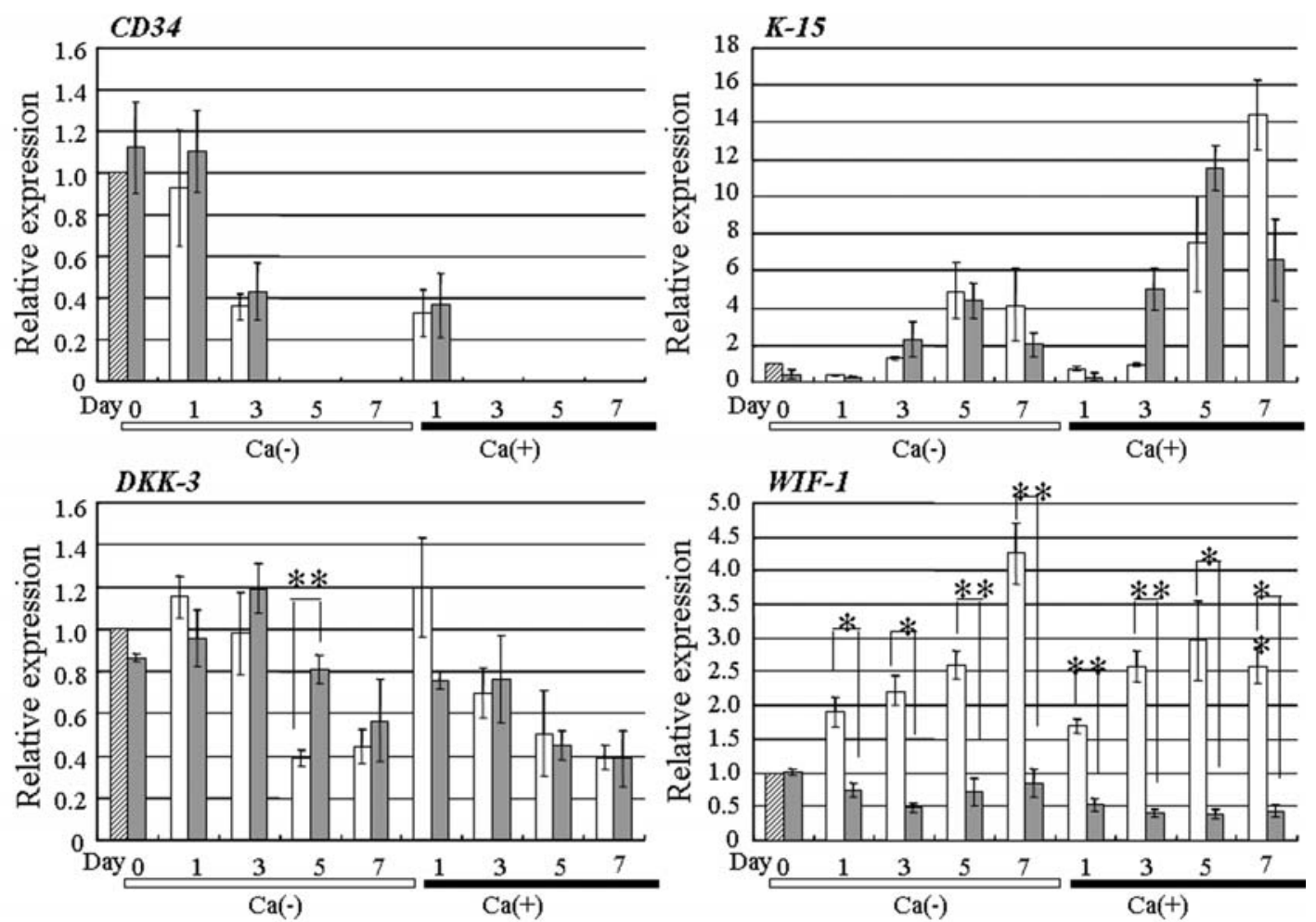

Figure 7. Real-time PCR analysis of $C D 34, K-15, D K K-3$ and $W I F-1$ expression in BDKs and NHEKs. Expression levels of $C D 34$ and $K-15$, reported as bulge stem cell markers, and $D K K-3$ and WIF-1, Wnt signaling inhibitors, were normalized to the levels of $A T P 5 F 1$. Expression levels in NHEKs on day 0 were set as 1.0 (shaded columns). White and gray columns indicate NHEKs and BDKs, respectively. Each column shows the average of three independent experiments. Bar, I SD. ${ }^{*} \mathrm{p} \leq 0.05 .{ }^{* *} \mathrm{p} \leq 0.01$.

both genes were lower in BDKs than NHEKs (Fig. 6A). The expression levels of ITG- $\alpha 6$ on day 7 with $\mathrm{CaCl}_{2}$ treatment was significantly higher in BDKs than in NHEKs $(\mathrm{p}<0.01)$ (Fig. 6A). These results showed that changes of expression of prickle cell layer marker genes were smaller in BDKs than NHEKs.

We examined five stem cell markers, CD34, CD200, Oct3/4, SOX2, and NANOG, in BDKs and NHEKs after $\mathrm{CaCl}_{2}$ treatment. Without $\mathrm{CaCl}_{2}$ treatment, expression of CD34 was positive until day 3 and then negative on days 5 and 7 in both BDKs and NHEKs (Fig. 6B). With $\mathrm{CaCl}_{2}$ treatment, $C D 34$ was expressed on day 1 only in BDKs and NHEKs (Fig. 6B). Expression of CD200, reported as a more specific bulge stem cell marker than CD34, was positive in NHEKs on day 0 and the expression levels were stable with or without $\mathrm{CaCl}_{2}$ until day 7. In BDKs, the expression levels of $C D 200$ were similar to those of NHEKs without $\mathrm{CaCl}_{2}$ until day 7 and with $\mathrm{CaCl}_{2}$ until day 5. But on day 7 with $\mathrm{CaCl}_{2}$, significantly higher expression of $C D 200$ was observed in BDKs than in NHEKs $(\mathrm{p}<0.05)$. OCT3/4 levels were similar in both BDKs and NHEKs without $\mathrm{CaCl}_{2}$ treatment. In the presence of $\mathrm{CaCl}_{2}$, expression of OCT3/4 was slightly increased until day 5 but decreased on day 7 in both cells (Fig. 6B). The expression of $S O X 2$ decreased on day 7 without $\mathrm{CaCl}_{2}$ treatment in both cells. In the presence of $\mathrm{CaCl}_{2}, \mathrm{SOX} 2$ levels in NHEKs was significantly lower than those in BDKs on day 7 ( $\mathrm{p}<0.01)$. NANOG levels showed some increase during incubation in the absence of $\mathrm{CaCl}_{2}$ in both BDKs and
NHEKs. In the presence of $\mathrm{CaCl}_{2}, N A N O G$ levels increased until day 5 and then decreased on day 7 in both cells with expression levels in NHEKs slightly lower than in BDKs $(\mathrm{p}=0.05)$. These data suggest that BDKs may be refractory to differentiation.

We then examined expression levels of $C D 34$ and $K-15$ using real-time PCR. In both BDKs and NHEKs, CD34 expression decreased rapidly to be essentially undetectable on day 5 (Fig. 7). K-15 expression was low in both cells in the absence of $\mathrm{CaCl}_{2}$ on days 0 and 1 , and significantly increased on days 3-7. In the presence of $\mathrm{CaCl}_{2}, \mathrm{~K}-15$ levels were highly elevated on days 3-7 (Fig. 7). These results indicate that $K-15$ was one of the differentiation markers of the BDKs and NHEKs, and not suitable to detect bulge-stem cells in culture.

We also examined $D K K-3$ and $W I F-1$ genes involved in the Wnt signaling pathway. In the absence of $\mathrm{CaCl}_{2}, D K K-3$ levels were similar in NHEKs and BDKs except day 5 when NHEKs showed significantly lower levels than BDKs $(\mathrm{p}<0.01)$. $\mathrm{CaCl}_{2}$ treatment suppressed $D K K-3$ expression to a similar extent in both cell types. In the absence of $\mathrm{CaCl}_{2}, W I F-1$ expression remained low in BDKs, but in NHEKs WIF-1 levels were elevated 2 -fold on day 1 and 4 -fold on day 7 , resulting in higher WIF-1 expression levels in NHEKs than in BDKs ( $<<0.05$ on days 1 and 3, p<0.01 on days 5 and 7). Similarly, in the presence of $\mathrm{CaCl}_{2}, W I F-1$ expression became $50 \%$ in BDKs on day 1 and remained low until day 7 , while in NHEKs, WIF-1 expression level was 2- to 3-fold on 
days 1-7, resulting in much higher $W I F-1$ expression levels in NHEKs ( $p<0.01$ on days 1,3 and $7, p<0.05$ on day 5$)$.

\section{Discussion}

Primary culture and characteristics of BDK cells. We report the cultivation of BDKs not using invasive skin biopsies but from hairs plucked from donors. With the culture method used, hair specimens from all 43 donors yielded BDKs with an average PO rate of $47 \%$, regardless of age and gender of the donor. BDKs were also obtained from atopic donors with the average PO rate of $41 \%$, indicating that BDKs can be established from donors with variety of backgrounds. Interestingly, three hairs of a 76-year old subject with alopecia areata also yielded BDKs with the number of population doublings, the population doubling time, the total cell yields, and morphology of this donor's BDKs similar to those of younger donor's BDKs.

The expression of $C D 34$, one of the stem cell markers, was positively detected in cells in primary outgrowth and in early passages (Fig. 4B). However, immunostaining failed to detect CD34-positive cells in the primary outgrowth, suggesting low levels of CD34 expression and/or very few CD34-positive cells in the primary outgrowth. Only 1-2\% of cells separated directly from human bulges have been reported to be CD34-positive by FACS analysis (25). Positive $C D 34$, high proliferation ability, and ageindependent growth of BDKs strongly suggest that BDKs are derived from the stem cell fraction existing in bulge regions.

From one hair follicle, $2 \times 10^{11}-9 \times 10^{11} \mathrm{BDKs}$ were obtained and the life span in culture was at least 3 months (Fig. 3 and Table II). Hence, total cell yield was $4.0 \times 10^{7}-1.8 \times 10^{8}$ times the number of cells in passage 1. In comparison of growth between BDKs with NHEKs, we found BDKs showed less total cell yield and fewer number of population doublings than NHEKs. If CD34-positive cells constitute $1-2 \%$ cells in a bulge as reported (25), only 50-100 stem cells might exist in $\sim 5000$ BDKs in primary outgrowth. Since 50-100 cells divided at least 6 times to reach 5000 cells, 5000 cells used for the first passage were already in six population doublings. Consequently, the number of population doublings and other cell proliferation ability of BDKs may be the same or better than NHEKs, supporting the possibility that our BDKs were derived from bulge stem cells.

Gene expression of BDKs during differentiation. After induction of differentiation with $\mathrm{CaCl}_{2}$, the reduction of CD34 expression was observed in both NHEKs and BDKs in a similar manner. Morphological changes and decreased expression of $K-5$, one of the basal cell markers, were also observed in both cell types. However, lower induction of $K-1$ and $K-10$, prickle cell layer markers, and smaller reduction of expression of $I T G-\alpha 6$, a basal cell layer marker, were detected in BDKs than in NHEKs (Fig. 6A). Expression of $K-15$, reported as a bulge-stem cell marker, was significantly induced in both NHEKs and BDKs after $\mathrm{CaCl}_{2}$ treatment (Figs. 6A and 7). Immunostaining also showed that higher expression of $\mathrm{K}-15$ in both cells after $\mathrm{CaCl}_{2}$ treatment (data not shown). We analyzed CD200 in BDKs which has been reported to be a more specific bulge stem cell marker than CD34 (8). CD200 levels in BDKs were found to be higher after $\mathrm{CaCl}_{2}$ treatment (Fig. 6B), suggesting that $C D 200$, like $K-15$, was not a suitable marker for bulge stem cells, at least in our culture condition. $C D 200$ was selected as one of bulge stem cell marker genes by cDNA microarray analysis of K-15positive cells in the bulge region that were isolated from skin biopsy specimens by microdissection, trypsinization, and FACS sorting (8). Since these procedures may alter characteristics of cells, gene expression profiles in such cells may differ from those in BDKs obtained by our culture method which might preserve more physiological conditions.

We also analyzed several other genes reported as specific to bulge stem cells. Expression of SOX2 and NANOG was higher in $\mathrm{BDKs}$ than in NHEKs after $\mathrm{CaCl}_{2}$-induced differentiation (Fig. 6B), suggesting that BDKs are more resistant to differentiation than NHEKs.

Recently, active Wnt signaling has been considered to be important to maintain embryonic and adult stem cells and cancer stem cells $(23,28)$. Wnt signaling is also active in basal cell carcinomas of the skin (29). Since high expression of two Wnt signaling-inhibitor genes, $D K K-3$ and WIF-1, has been detected in bulge stem cells (8), we examined expression of these genes in BDKs and NHEKs. Lower expression of $D K K-3$, also called $R E I C$, is detected in many human cancers than in normal tissues $(30,31)$. Reduced $D K K-3$ expression occurred as an early event of tumor development (32), and its overexpression induced apoptosis in tumor cells (33). Expression of $W I F-1$, wnt inhibitory factor-1, that directly binds Wnt proteins to inhibit Wnt signaling, was low in several solid tumors $(34,35)$.

$D K K-3$ was expressed in NHEKs and BDKs but expression levels decreased to half on day 5 after $\mathrm{CaCl}_{2}$ treatment in both cells (Fig. 7). WIF-1 expression was about the same in BDKs and NHEKs on day 0 and $\mathrm{CaCl}_{2}$ treatment increased 2-fold in NHEKs and decreased to half in BDKs on day 1. On day 3, expression levels further increased in NHEKs, but remained low in BDKs (Fig. 7), indicating that regulatory mechanisms of WIF-1 may be different between NHEKs and BDKs. It has been proposed that, in cancer cells, levels of $W I F-1$ are negatively regulated through hypermethylation in the promoter regions $(34,35)$. The possibility exists that methylation may be lost in NHEKs but maintained or induced in BDKs during differentiation.

Our results strongly suggest that BDKs are refractory to differentiation. BDKs have also been reported to play a role in wound healing (19) and development of psoriasis and cancer (36). Our data suggest that maintenance of active Wnt signaling pathway in BDKs through suppression of WIF-1 expression may be important for immediate response of BDKs to skin injury facilitating re-epithelization and for keeping undifferentiated features of BDKs that may be important to develop basal cell carcinoma of the skin. Our data also suggest that characteristics of BDKs, such as expression of stem cell markers, high proliferation capacity, and resistance to differentiation, are related to down-regulation of WIF-1 expression. Constitutional activation of Wnt signaling pathway in bulge stem cells may lead to carcinogenesis. Activation of WIF-1 expression may be a key for prevention of skin cancers originating from the bulge region. 


\section{Acknowledgements}

We thank Ms. A. Iemoto and Ms. Y. Sugihara for their technical assistance. This study was partially supported by a grant-in-aid from the Project for Frontier of Science (No. F980172) from the Ministry of Education, Science, Sports and Culture, Government of Japan.

\section{References}

1. Fuchs E: Scratching the surface of skin development. Nature 445: 834-842, 2007.

2. Cotsarelis G, Sun TT and Laver RM: Label-retaining cells reside in the bulge area of pilosebaceous unit: implications for follicular stem cells, hair cycle, and skin carcinogenesis. Cell 617: 1329-1337, 1990

3. Taylor G, Lehrer MS, Jensen PJ, Sun TT and Lavker RM: Involvement of follicular stem cells in forming not only the follicle but also the epidermis. Cell 102: 451-461, 2000.

4. Oshima H, Rochat A, Kedzia C, Kobayashi K and Barrandon Y: Morphogenesis and renewal of hair follicles from adult multipotent stem cells. Cell 104: 233-245, 2001.

5. Amoh Y, Li L, Katsuoka K, Penman S and Hoffman RM Multipotent nestin-positive, keratin-negative hair-follicle bulge stem cells can form neurons. Proc Natl Acad Sci USA 102: 5530-5534, 2005.

6. Hoffman RM: The potential of nestin-expressing hair follicle stem cells in regenerative medicine. Expert Opin Biol Ther 7: 289-291, 2007

7. Hoogduijn MJ, Gorjup E and Genever PG: Comparative characterization of hair follicle dermal stem cells and bone marrow mesenchymal stem cells. Stem Cells Dev 15: 49-60, 2006

8. Ohyama M, Terunuma A, Tock CL, et al: Characterization and isolation of stem cell-enriched human hair follicle bulge cells. J Clin Invest 116: 249-260, 2006.

9. Morris RJ, Liu Y, Marles L, et al: Capturing and profiling adult hair follicle stem cells. Nat Biotechnol 22: 411-417, 2004.

10. Trempus CS, Morris RJ, Bortner CD, et al: Enrichment for living murine keratinocytes from the hair follicle bulge with the cell surface marker CD34. J Invest Dermatol 120: 501-511, 2003.

11. Yu H, Fang D, Kumar SM, et al: Isolation of a novel population of multipotent adult stem cells from human hair follicles. Am J Pathol 168: 1879-1888, 2006.

12. Tumbar T, Guasch G, Greco V, et al: Defining the epithelial stem cell niche in skin. Science 303: 359-363, 2004.

13. Grinnell KL, Yang B, Eckert RL and Bickenbach JR: Dedifferentiation of mouse interfollicular keratinocytes by the embryonic transcription factor Oct-4. J Invest Dermatol 127: 260-262, 2007

14. Takahashi K, Tanabe $\mathrm{K}$, Ohnuki M, et al: Induction of pluripotent stem cells from adult human fibroblasts by defined factors. Cell 131: 861-872, 2007.

15. Hennings H, Michael D, Cheng C, Steinert P, Holbrook K and Yuspa SH: Calcium regulation of growth and differentiation of mouse epidermal cells in culture. Cell 19: 245-254, 1980

16. Yano S, Ito Y, Fujimoto M, Hamazaki TS, Tamaki K and Okochi H: Characterization and localization of side population cells in mouse skin. Stem Cells 23: 834-841, 2005.

17. Vellucci VF, Germino FJ and Reiss M: Cloning of putative growth regulatory genes from primary human keratinocytes by subtractive hybridization. Gene 166: 213-220, 1995.

18. Tennenbaum T, Belanger AJ, Quaranta V and Yuspa SH: Differential regulation of integrins and extracellular matrix binding in epidermal differentiation and squamous tumor progression. J Investig Dermatol Symp Proc 1: 157-161, 1996.
19. To M, Liu Y, Yang Z, et al: Stem cells in the hair follicle bulge contribute to wound repair but not to homeostasis of the epidermis. Nat Med 11: 1351-1354, 2005.

20. Krüger K, Blume-Peytavi U and Orfanos CE: Basal cell carcinoma possibly originates from the outer root sheath and/or the bulge region of the vellus hair follicle. Arch Dermatol Res 291: 253-259, 1999.

21. Affara NI, Trempus CS, Schanbacher BL, et al: Activation of Akt and mTOR in $\mathrm{CD} 34^{+} / \mathrm{K}_{1} 5^{+}$keratinocyte stem cells and skin tumors during multi-stage mouse skin carcinogenesis. Anticancer Res 26: 2805-2820, 2006.

22. Trempus CS, Morris RJ, Ehinger M, et al: CD34 expression by hair follicle stem cells is required for skin tumor development in mice. Cancer Res 67: 4173-4181, 2007

23. Malanchi I, Peinado H, Kassen D, et al: Cutaneous cancer stem cell maintenance is dependent on beta-catenin signalling. Nature 452: 650-653, 2008.

24. Moll I: Proliferative potential of different keratinocytes of plucked human hair follicles. J Invest Dermatol 105: 14-21, 1995.

25. Raposio E, Guida C, Baldelli I, et al: Characterization of multipotent cells from human adult hair follicles. Toxicol In Vitro 21: 320-323, 2007

26. Gho CG, Braun JE, Tilli CM, Neumann HA and Ramaekers FC: Human follicular stem cells: their presence in plucked hair and follicular cell culture. Br J Dermatol 150: 860-868, 2004.

27. Sasaki K, Tamura S, Tachibana H, et al: Expression and role of p27kip1 in neuronal differentiation of embryonal carcinoma cells. Brain Res Mol Brain Res 77: 209-221, 2000

28. Grigoryan T, Wend P, Klaus A and Birchmeier W: Deciphering the function of canonical Wnt signals in development and disease: conditional loss- and gain-of-function mutations of beta-catenin in mice. Genes Dev 22: 2308-2341, 2008.

29. Asplund A, Gry Björklund M, Sundquist C, et al: Expression profiling of microdissected cell populations selected from basal cells in normal epidermis and basal cell carcinoma. Br J Dermatol 158: 527-538, 2008.

30. Tsuji T, Miyazaki M, Sakaguchi M, Inoue Y and Namba M: A REIC gene shows down-regulation in human immortalized cells and human tumor-derived cell lines. Biochem Biophys Res Commun 268: 20-24, 2000.

31. Abarzua F, Sakaguchi M, Takaishi M, et al: Adenovirus-mediated overexpression of REIC/Dkk-3 selectively induces apoptosis in human prostate cancer cells through activation of c-Jun-NH2kinase. Cancer Res 65: 9617-9622, 2005.

32. Kuphal S, Lodermeyer S, Bataille F, Schuierer M, Hoang BH and Bosserhoff AK: Expression of Dickkopf genes is strongly reduced in malignant melanoma. Oncogene 25: 5027-5036, 2006.

33. Kashiwakura Y, Ochiai K, Watanabe M, et al: Down-regulation of inhibition of differentiation-1 via activation of activating transcription factor 3 and Smad regulates REIC/Dickkopf-3induced apoptosis. Cancer Res 68: 8333-8341, 2008.

34. Reguart N, He B, Xu Z, et al: Cloning and characterization of the promoter of human Wnt inhibitory factor-1. Biochem Biophys Res Commun 323: 229-234, 2004.

35. Taniguchi $\mathrm{H}$, Yamamoto $\mathrm{H}$, Hirata $\mathrm{T}$, et al: Frequent epigenetic inactivation of Wnt inhibitory factor-1 in human gastrointestinal cancers. Oncogene 24: 7946-7952, 2005

36. Kamstrup M, Faurschou A, Gniadecki R and Wulf HC: Epidermal stem cells - role in normal, wounded and pathological psoriatic and cancer skin. Curr Stem Cell Res Ther 3: 146-150, 2008. 